\title{
Omega-3 Polyunsaturated Fatty Acids, Metabolic Syndrome and Diabetes Mellitus
}

\author{
Victoria Serhiyenko*, Ludmila Serhiyenko and Alexandr Serhiyenko \\ Department of Endocrinology, Danylo Galytsky Lviv National Medical University, Ukraine
}

Submission: January 22, 2018; Published: February 06, 2018

*Corresponding author: Victoria Serhiyenko, Department of Endocrinology, Danylo Halytsky Lviv National Medical University, Ukraine, 69, Pekarska str., 79010, Ukraine; Tel: +38-322-76-94-96, E-mail: 965554@ukr.net; serhiyenkoa@gmail.com

\begin{abstract}
Omega-3 polyunsaturated fatty acids ( $\omega-3$ PUFAs) are increasingly being used to prevent cardiovascular diseases (CVD), and cardiac societies recommend the intake of $1 \mathrm{~g} /$ day of the two $\omega$-3 PUFAs eicosapentaenoic and docosahexaenoic acid for primary and secondary prevention of CVD. Clinical trials clearly suggest beneficial effects of $\omega$-PUFAs consumption on lipid metabolism profile, their anti-inflammatory actions; on endothelial activation, which are likely to improve vascular function; antithrombotic and antiatherosclerotic properties. Experimental studies demonstrate direct antiarrhythmic effects, which have been challenging to document in humans. By targeting arterial stiffness and endothelial dysfunction administration of $\omega$-3 PUFAs may prevent atherosclerosis and CVD development. A synergistic interplay showed by $\omega$-3 PUFAs prescription suggest the potential to beneficially impact on fundamental steps involved in the development of preclinical atherosclerosis. We reviewed available evidence of the benefits of $\omega$-PUFAs administration, especially to patients with CVD, metabolic syndrome and type 2 diabetes mellitus, including their effects on potential molecular pathways, effects on glucose and lipids metabolism parameters, thrombocyte aggregation parameters and haemostasis, endothelial function, antioxidant/anti-inflammation and antiarrhythmic properties.
\end{abstract}

Keywords: Omega-3 polyunsaturated fatty acids; Coronary heart disease, atherosclerosis; Diabetes mellitus; Glucose, lipids; Inflammation; Platelets; Haemostasis; Endothelium; Heart rate variability; Arrhythmias; Arterial stiffness

Abbreviations: $\omega-3$ and $\omega-6$ PUFAs: $\Omega-3$ and $\omega-6$ Polyunsaturated Fatty Acids; MetS: Metabolic Syndrome; T2DM: Type 2 Diabetes Mellitus; CVD: Cardiovascular Diseases; DLP: Dyslipoproteinemia; OS: Oxidative Stress

\section{Introduction}

Numerous studies report salutary effects of $\omega-3$ polyunsaturated fatty acids ( $\omega$-PUFAs), i.e. eicosapentaenoic (EPA) and docosahexaenoic acid (DHA) on cardiovascular diseases (CVD) risk factors. These effects include lowering of serum triglyceride (TG) by reducing of hepatic TG production; lowering of blood pressure (BP) by improving of endothelial cell functution; decreasing of platelet aggregation by reducing of prothrombotic prostanoids; decreasing inflammation via reduction in 4-series leukotrienes (LT) production; protection from arrhythmias by modulation of electrophysiological properties of cardiac myocytes. Systematic meta analysis suggests that high doses of $\omega-3$ PUFAs $(\sim 3 \mathrm{~g} /$ day $)$ produce a small, but significant decrease in systolic blood pressure (SBP) in older and hypertensive subjects [1,2]. The aim of this study was to review the latest evidence about the $\omega$-PUFAs, metabolic syndrome (MetS) and type 2 diabetes mellitus (T2DM).

\section{Discussion}

$\Omega-3$ and $\omega-6$ PUFAs are essential fatty acids, as they cannot be synthesized de novo in humans. There are limited data available regarding the exact amount of dietary $\omega-3$ PUFAs consumed by the general population. It is reported that the total daily intake of dietary $\omega-3$ PUFAs in the US is approximately 1.6g. Of this $\alpha$-linolenic acid ( $\alpha$-LLA) accounts for approximately $1.4 \mathrm{~g} / \mathrm{q} . \mathrm{d}$, and only $0.1-0.2 \mathrm{~g} / \mathrm{q}$.d. comes from EPA and DHA. The conversion rate from $\alpha$-LLA to EPA and DHA is variable (0.2-15\%). Therefore, in general, the total amount of EPA and DHA available to the body from current dietary patterns is well below the recommended amounts. EPA and DHA didn't show a significant negative effect on glucose metabolism [3].

Several experimental studies have shown that long-chain $\omega$-PUFAs inhibit the absorption of cholesterol in the intestine and its synthesis in the liver, lead to increased clearance of 


\section{Current Research in Diabetes \& Obesity Journal}

lipoproteins in the blood, prevent the development of insulin resistance (IR) in experimental diabetes, increase the level of glucose transporter 4 in skeletal muscles, have a positive effect on age related decrease of blood flow in the brain and improve glucose utilization under stress; there isn't any influence on the development of hypertension (HT) and MetS. $\Omega$-3 PUFAs decrease level of BP, dose-dependent prevent the development of T2DM, IR, contribute to positive changes of blood coagulation parameters; enhance endothelial cell migration and inhibits the proliferation of smooth muscle cells [4]. A meta-analysis of 18 studies found a significant effect of fish oil to lower TG concentrations and increase high-density lipoprotein cholesterol (HDL-C) in the blood; while there were no statistically significant changes in preprandial glucose, glycated hemoglobin A1c, total cholesterol, low density-lipoprotein cholesterol levels. $\Omega$-3 PUFAs may affect the IR and glucose homeostasis by inhibition of IR in the muscle tissue >adipose tissue >>liver, inhibition of insulin secretion, which defer the development of T2DM; and on the state of lipid metabolism (in particular, reduce the concentration of TG, very low density-lipoprotein cholesterol (VLDL-C), increase of HDL-C, improve lipid profile by mixed hyperlipidaemia (HLP), slightly decrease BP, improve endothelial function, have an positive impact on the antioxidant status and inflammatory reactions [5]. ת-3 PUFAs decrease VLDL assembly and secretion, resulting in diminished TG production, through a decreased sterol receptor element binding protein-1c activity [6,5].

The highly concentrated pharmaceutical preparation Omacor $^{\mathrm{TM}}$ (Pronova Biocare, Lysaker, Norway), known as Lovaza $^{\mathrm{TM}}$ (Glaxo Smith Kline, St Petersberg, FL, US) in North America is approved by the FDA as an adjunct to diet to reduce very high TG levels ( $\left.\geq 500 \mathrm{mg} \bullet \mathrm{dL}^{-1}\right)$ in adults. Each 1 -g capsule of $\omega$-3-acid ethyl esters contains ethyl esters of EPA (0.465 g) and DHA (0.375g). Patients take a q.d. dose of 4-g or two 2-g doses (two capsules b.i.d.) [7]. Clinical trials have shown that administration of $4{\mathrm{~g} \bullet \mathrm{day}^{-1} \text { of Lovaza }}^{\mathrm{TM}}$ results in a decrease in TG levels of 30-50\%; does not affect the efficacy of statins [8,5]. In patients with combined HLP, co-administration of Lovaza ${ }^{\mathrm{TM}}$ with statins was a safe and effective means of lowering serum TG, despite the persistent high TG levels when the patients received statins alone $[9,5]$.

The anti-inflammatory actions of marine $\omega$-3 PUFAs are [10]: reduced leucocyte chemotaxis (via decreased production of some chemoattractants (e.g. leukotriene B4 down-regulated expression of receptors for chemoatttactants); reduced adhesion molecule expression and decreased leucocyte-endothelium interaction (via down-regulated expression of adhesion molecule genes [via the nuclear factor kappa $\mathrm{B}(\mathrm{NF}-k \mathrm{~B})$ (i.e. peroxisome proliferator-activated receptor- $\gamma$ (PPAR- $\gamma$ ) etc.); decreased production of eicosanoids from arachidonic acid (AA) (via lowered membrane content of AA; inhibition of AA metabolism); decreased production of AA containing endocannabinoids (via lowered membrane content of AA); increased production of 'weak' eicosanoids from EPA (via increased membrane content of EPA); increased production of anti-inflammatory EPA and DHA containing endocannabinoids (via increased membrane content of EPA and DHA); increased production of pro-resolution resolvins and protectins (via increased membrane content of EPA and DHA); decreased production of inflammatory cytokines (via down-regulated expression of inflammatory cytokine genes (via NF- $k$ B, i.e. PPAR- $y$ etc.); decreased T cell reactivity (via disruption of membrane rafts (via increased content of EPA and DHA in specific membrane regions).

$\Omega$-3 PUFAs may decrease the risk of atherothrombosis by affecting platelet aggregation and haemostasis. The antithrombotic properties of EPA and DHA have been attributed to the incorporation into platelet phospholipids at the expense of the $\omega-6$ PUFAs, such as AA. An important set of pathways clearly influenced by changes in the $\omega-3 / \omega-6$ ratio are those for synthesis of eicosanoids. These include the cyclooxygenase (COX), lipoxygenase and cytochrome P450 epoxygenase pathways, for which EPA and DHA compete with AA as a substrate, inhibiting the production of the proaggregatory thromboxane A2 (TXA2) originating from AA. Indeed, the production of TXA2 from platelets stimulated by a variety of agonists decreased by between $60 \%$ and $80 \%$ after fatty acid supplementation both in vitro and in vivo $[11,5]$. The mechanism by which $\omega-3$ PUFAs influence endothelial function is mediated by their incorporation into biological membrane phospholipids; this allows modulation of membrane composition and fluidity. The reason lies in the fact that endothelial cell membrane houses caveolae and lipid rafts where several receptors and signaling molecules crucial for cell function are concentrated [12]. Caveolae-associated receptormediated cellular signal transduction includes important pathways such as the, the nitric oxide (NO)/cyclic guanosine monophosphate signaling pathway, the nicotinamide adenine dinucleotide phosphate oxidase and tumor necrosis factor- $\alpha$ / NF- $k$ B induced COX- 2 and prostaglandin E2 activation pathway. By modulating the composition of caveolae, as described for other classes of lipids $\omega-3$ PUFAs may exert their beneficial effects, which include increased NO production and reduced production of proinflammatory mediators $[13,12]$. In addition to increasing NO production, $\omega-3$ PUFAs decrease oxidative stress.

The incorporation of $\omega-3$ PUFAs in synaptic membranes could potentially influence the autonomic control of the heart. Both nervous tissue and heart tissue have a high content of $\omega$-3 PUFAs (especially DHA) and this may be consistent with the finding that this marine $\omega-3$ PUFAs may modulate cardiac autonomic function as assessed by heart rate variability (HRV) [14]. Thus, $\omega-3$ PUFAs may modulate HRV both at the level of the autonomic nervous system and the heart. Most of the data support that $\omega-3$ PUFAs beneficially modulates cardiac autonomic control thereby possibly reducing the risk of arrhythmias. Accumulating evidence from in vivo and in vitro experiments has demonstrated that $\omega$-3 PUFAs exert antiarrhythmic effects through modulation of myocyte electrophysiology. $\Omega$-3 PUFAs reduce the activity of membrane $\mathrm{Na}+$ channels in cardiomyocytes, thus increasing the 


\section{Current Research in Diabetes \& Obesity Journal}

threshold for membrane potential depolarization. EPA and DHA also modulate the activity of L-type $\mathrm{Ca}^{2+}$ channels, leading to a reduction in free cytosolic $\mathrm{Ca}^{2+}$ ion, which stabilizes myocyte electrical excitability to prevent fatal arrhythmia. EPA blocks the $\mathrm{Na}^{+} / \mathrm{Ca}^{2+}$ channel; however, a single amino-acid point mutation in this channel attenuated the inhibitory effect of EPA. These findings suggested that the cardioprotective effect of $\omega-3$ PUFAs is mediated by direct interaction with membrane ion channels [15].

$\Omega$-3 PUFAs intake has shown to reduce BP especially in HT by interacting with several mechanisms of BP regulation: reduction of stroke volume and heart rate; improvement of left ventricular (LV) diastolic filling; reduction of peripheral vascular resistances; improvement of endothelial-dependent and endothelial-independent vasodilation (stimulation of NO production; reduction of the asymmetric di-methyl-arginine; reduction of endothelin-1; relaxation of vascular smooth muscle cells; metabolic effects on perivascular adipocytes; endothelial regeneration. Mechanisms of HT-related organ damage protection: anti-inflammatory, antioxidant, and antithrombotic effects; reduction of arterial stiffness; experimental effects on LV hypertrophy and abnormal gene expression; effects on atherosclerotic plaque progression and stability [7]. $\Omega-3$ PUFAs offer a scientifically supported means of reducing arterial stiffness and this may account for some of the purported cardioprotective effects of $\omega-3$ PUFAs $[16,17]$.

\section{Conclusion}

The antiarrhythmic effects of $\omega-3$ PUFAs, which occur by blocking various ion channels, are encouraging. So, cardiovascular benefits of $\omega-3$ PUFAs $[7,18]$ are: antidysrhythmic effects (reduced sudden death; possible prevention of atrial fibrillation; possible protection against pathologic ventricular arrhythmias; improvement in HRV; antiatherogenic effects (reduction in nonHDL-C levels; reduction in TG and VLDL-C levels; reduction in chylomicrons; reduction in VLDL and chylomicron remnants; increase in HDL-C levels; plaque stabilization; antithrombotic effects (decreased platelet aggregation; improved blood rheologic flow); anti-inflammatory and endothelial protective effects (reduced endothelial adhesion molecules and decreased leukocyte adhesion receptor expression; reduction in proinflammatory eicosanoids and LT's; vasodilation); decreased SBP and diastolic BP. Thus, further research to understand the mechanism of action and confirm the beneficially effect of $\omega-3$ PUFAs on BP profile, artery stiffness and HRV parameters in patiens with MetS, T2DM is needed.

\section{References}

1. Bonafini S, Antoniazzi F, Maffeis C, Minuz P, Fava C (2015) Beneficial effects of omega-3 PUFA in children on cardiovascular risk factors during childhood and adolescence. Prostaglandins Other Lipid Mediat 120: 72-79.

2. Jeppesen C, Schiller K, Schulze MB (2013) Omega-3 and omega-6 fatty acids and type 2 diabetes. Curr Diab Rep 13(2): 279-288.

3. Kandasamy N, Joseph F, Goenka N (2008) The role of omega-3 fatty acids in cardiovascular disease, hypertriglyceridaemia and diabetes mellitus. Br J Diabet Vasc Dis 8(3): 121-128.

4. Von Schacky C (2010) Omega-3 fatty acids vs. cardiac disease: the contribution of the omega-3 index. Cell Mol Biol 56(1): 93-101.

5. De Roos B, Mavrommatis Y, Brouwer IA (2009) Long-chain n-3 polyunsaturated fatty acids: new insights into mechanisms relating to inflammation and coronary heart disease. Br J Pharmacol 158(2): 413-428.

6. Jump DB (2008) N-3 polyunsaturated fatty acid regulation of hepatic gene transcription. Curr Opin Lipidol 19(3): 242-247.

7. Bradberry JC, Daniel E, Hilleman DE (2013) Overview of omega-3 fatty acid therapies. P T 38(11): 681-691.

8. McKenney JM, Swearingen D, Di Spirito M, Doyle R, Pantaleon C, et al. (2006) Study of the pharmacokinetic interaction between simvastatin and prescription omega-3-acid ethyl esters. J Clin Pharmacol 46(7): 785-791.

9. Davidson MH, Stein EA, Bays HE, Maki KC, Doyle RT, et al. (2007) COMBination of prescription omega with simvastatin (COMBOS) investigators. Efficacy and tolerability of adding prescription omega-3 fatty acids $4 \mathrm{~g} / \mathrm{d}$ to simvastatin $40 \mathrm{mg} / \mathrm{d}$ in hypertriglyceridemic patients: an 8-week, randomized, double-blind, placebo-controlled study. Clin Ther 29(7): 1354-1367.

10. Calder PC (2015) Marine omega-3 fatty acids and inflammatory processes: Effects, mechanisms and clinical relevance. Biochim Biophis Acta 1851(4): 469-484

11. Kristensen SD, Schmidt EB, Dyerberg J (1989) Dietary supplementation with n-3 polyunsaturated fatty acids and human platelet function: a review with particular emphasis on implications for cardiovascular disease. J Intern Med Suppl 731: 141-150.

12. Williams JL, Palmer TM (2014) Cavin-1: caveolae-dependent signalling and cardiovascular disease. Biochem Soc Trans 42(2): 284-288.

13. Rauch B, Schiele R, Schneider S, Gohlke H, Diller F, et al. (2006) Omega-study group. highly purified omega-3 fatty acids for secondary prevention of sudden cardiac death after myocardial infarction-aims and methods of the OMEGA-study. Cardiovasc Drugs Ther 20(5): 365375 .

14. Christensen $H$ (2015) Omega-3 polyunsaturated fatty acids and heart rate variability. Front Physiol 2: 84.

15. Endo J, Arita M (2016) Cardioprotective mechanism of omega-3 polyunsaturated fatty acids. J Cardiol 67(1): 22-27.

16. Koumaras C, Katsiki N, Athyros VG, Karagiannis A (2013) Metabolic syndrome and arterial stiffness: the past, the present and the future. J Cardiovasc Med (Hagerstown) 14(10): 687-689.

17. Serhiyenko VA, Serhiyenko AA (2015) Diabetic cardiac autonomic neuropathy: Do we have any treatment perspectives? World J Diabetes 6(2): 245-258.

18. Serhiyenko VA, Serhiyenko AA (2018) Cardiac autonomic neuropathy: Risk factors, diagnosis and treatment. World J Diabetes 9(1): 1-24. 


\section{Current Research in Diabetes \& Obesity Journal}

(C) This work is licensed under Creative

BY DOI: 10.19080/CRDOJ.2018.05.555670
Your next submission with Juniper Publishers will reach you the below assets

- Quality Editorial service

- Swift Peer Review

- Reprints availability

- E-prints Service

- Manuscript Podcast for convenient understanding

- Global attainment for your research

- Manuscript accessibility in different formats

( Pdf, E-pub, Full Text, Audio)

- Unceasing customer service

Track the below URL for one-step submission https://juniperpublishers.com/online-submission.php 